\section{§11. Ion Temperature Gradient Modes in Toroidal Helical Systems}

Kuroda, T. (Grad. Univ. for Adv. Studies), Sugama, H., Kanno, R., Okamoto, M.

The ion temperature gradient (ITG) mode is one of the drift wave instabilities, which is considered a cause of the anomalous ion heat transport in high temperature core regions of tokamak plasmas. Recently, LHD succeeded in obtaining high ion temperature, $T_{i}(0) \geq$ $3 \mathrm{keV}$, by means of neutral beam injection. Observed ion temperature profiles are steeper than the density profiles. Thus, ITG modes are expected to become unstable and to drive anomalous transport in helical systems as well. Helical systems have non-uniformity of the magnetic field (toroidicity and helical ripples) which can be expressed for a large aspect ratio case as

$$
B / B_{0}=1-\epsilon_{t} \cos \theta-\epsilon_{h} \cos (L \theta-M \zeta),
$$

where $\theta$ and $\zeta$ denote the poloidal and toroidal angles, and $L$ and $M$ represent the poloidal and toroidal period numbers. Here, $\epsilon_{t} \propto r$ and $\epsilon_{h} \propto r^{L}$ are assumed with the minor radius $r$. Then, drift motions of particles are driven by the gradient of the magnetic field strength and by the curvature of the magnetic field line, $\boldsymbol{v}_{d}=\Omega_{i}^{-1}\left(v_{\|}^{2}+v_{\perp}^{2} / 2\right) \boldsymbol{B} \times B^{-2} \nabla B$ where $\Omega_{i}$ is the ion gyrofrequency . The drift motions driven by both toroidicity and helical ripples affect the ITG mode in toroidal helical systems, in contrast with the tokamak ITG mode which is driven by only toroidicity.

In this work, we investigate the linear stability of electrostatic ITG modes. The Boltzmann distribution of electrons is assumed in response to the electrostatic perturbation $\phi$. The collisionless gyrokinetic equation of ions is used to describe the ion dynamics. Then, the dispersion relation is determined by the quasineutrality condition. We consider a large aspect-ratio and low $\beta$ system with magnetic field geometry (1) and use the ballooning representation to solve the parallel structure of $\phi$ and to obtain the dispersion relation, numerically. The perpendicular structure is represented by the wavenumber vector $k_{\perp}=k_{\alpha}\left(\nabla \alpha+\theta_{k} \nabla q\right)$ where $q$ is the safety factor, $\alpha=\zeta-q \theta$ is the label for magnetic field line, and $k_{\alpha}=-n$ represents the toroidal mode number, which is related to the poloidal wavenumber as $k_{\theta}=n q / r$. The resultant dispersion relation is represented as an integral equation of the form

$$
\left(1+\frac{T_{e}}{T_{i}}\right) \phi(\theta, \omega)=\int_{-\infty}^{+\infty} \frac{d \theta^{\prime}}{\sqrt{2 \pi}} K\left(\theta, \theta^{\prime}\right) \phi\left(\theta^{\prime}, \omega\right),
$$

where $T_{e}$ and $T_{i}$ are the temperatures of electrons and ions, respectively, and $\theta$ is regarded as a parallel coordinate variable. The integral kernel $K$ depends on parameters such as $\eta_{i}=\left[d\left(\ln T_{i}\right) / d r\right] /\left[d\left(\ln n_{i}\right) / d r\right], \epsilon_{n}=$ $\left[d\left(\ln n_{i}\right) / d r\right]^{-1} / R_{0}, \tau_{e}=T_{e} / T_{i}$, and $\hat{s}=(r / q)(d q / d r)[1]$.

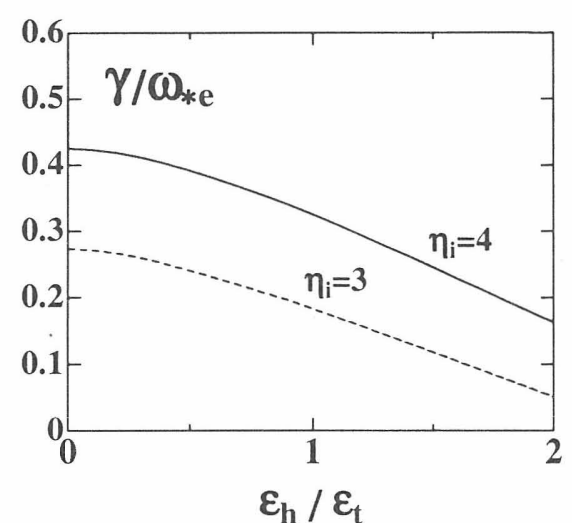

Figure 1: Normalized growth rate as a function of $\epsilon_{h} / \epsilon_{t}$.

The boundary conditions $\phi(\theta \rightarrow \pm \infty)=0$ are used. The complex-valued eigenfrequencies $\omega$ and eigenfunctions $\phi$ of the ITG mode for the helical systems are obtained by numerically solving Eq.(2). Then, the eigenfrequency $\omega$ is written as a function of several plasma parameters by

$$
\omega=\omega\left(q, \hat{s}, \theta_{k}, \alpha, k_{\theta}, \eta_{i}, \epsilon_{n}, \tau_{e}, \epsilon_{h} / \epsilon_{t}, L, M\right) .
$$

Standard parameters we used here are $q=2, \hat{s}=-1$ (negative shear), $\theta_{k}=0, \alpha=0, k_{\theta} \rho_{T i}=0.65, \eta_{i}=3$, $\epsilon_{n}=0.3, \tau_{e}=1, \epsilon_{h} / \epsilon_{t}=1, L=2$ and $M=10$. The $\epsilon_{h} / \epsilon_{t}$-dependence of the growth rate is shown in Fig. 1. With increasing $\epsilon_{h} / \epsilon_{t}$, the fine scale spatial structure of the helical ripples appear and good curvature regions come into existence on the outside of torus. Then, the parallel spatial structure of eigenfunction $\phi$ becomes oscillatory and the corresponding small phase velocity enhances the Landau damping. These effects result in reducing the growth rate. The $\alpha$-dependence is not so obvious as the stabilizing effects of varying the ballooning parameter $\theta_{k}$. Real part of the three dimensional eigenfunction $\phi$ for the standard parameters is shown as in Fig. 2 with using the ballooning transformation for a small radial region, $1.8<q<2.2$, where $q(r+\Delta r) \simeq q(r)+(\hat{s} q / r) \Delta r$ is used.

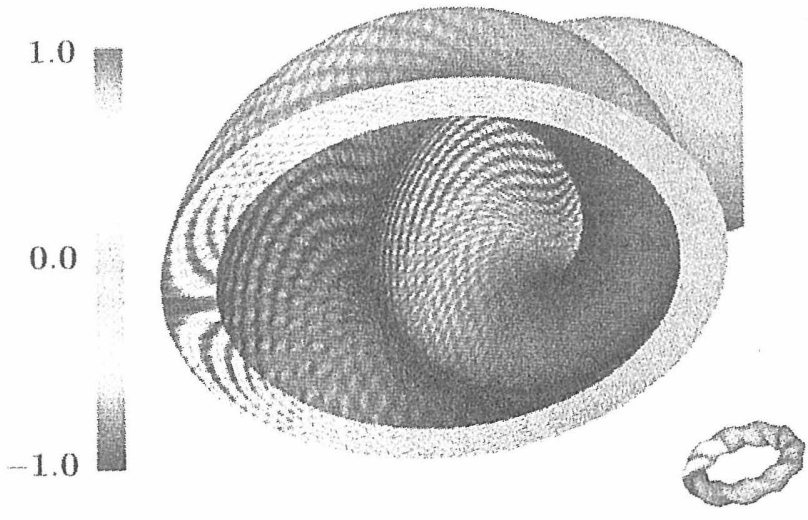

Figure 2: The 3D distribution of the eigenfunction $\phi$.

Reference

[1] T.Kuroda, H.Sugama, R.Kanno, M.Okamoto, to be published in J. Phys. Soc. Jpn.(2000). 